\section{Conditions expérimentales}

Les anticorps monoclonaux anti-MHC produits dans le cadre du projet Noé ont tous été testés sur différents muscles de dinde par immunofluorescence : les coupes réalisées au cryostat ont été fixées à l'acétone à $4{ }^{\circ} \mathrm{C}$ pendant 30 minutes puis incubées avec l'anticorps primaire dilué au $1 / 10$ et $2 \%$ de BSA pendant 1 heure à $37{ }^{\circ} \mathrm{C}$ puis lavés dans du PBS pH 7,2. Les coupes ont ensuite été incubées à la température de la pièce avec l'anticorps secondaire lié au FITC (Euromedex AP $160 \mathrm{~F})$ dilué au $1 / 100$ pendant 1 heure. Lorsque le signal paraissait prometteur, un contrôle à une dilution de l'anticorps primaire à $1 / 100$ a été réalisé.

Afin de fixer avec précision le type de chaque anticorps, chacun d'entre eux a été utilisé sur 4 muscles coupés en congélation : le pectoral (pectoralis major), composé presque exclusivement de fibres de type IIB, le PLD (posterior latissimus dorsi), composé d'un mélange de fibres de type IIA et IIB, l'ITC (iliotibialis cranialis), formé d'une zone hétérogène composée de fibres de type I, IIA et IIB et d'une zone hétérogène composée de fibres de type IIA et IIB, et l'ALD (anterior latissimus dorsi), composé de fibres de type III.

\section{Résultats}

Les résultats suivants ont été obtenus :

- 1 anticorps a fourni un marquage très faible des fibres squelettiques mais il marquait très fortement les artères présentes sur la coupe (MHC spécifique de fibre musculaire lisse) ;
- 32 anticorps ont fourni un signal nul ou faible ;

- 1 anticorps un signal très faible sur des fibres de type III (lentes) ;

- 16 anticorps un signal positif sur les fibres de type II, sans les différencier ;

- 5 anticorps pan-myosine (c'est-à-dire couvrant toutes les isoformes de myosine), marquage de toutes les fibres ;

- 3 pan-myosine avec un signal supérieur pour les fibres de type I (lentes);

- 3 pan-myosine avec un signal supérieur pour les fibres de type II (rapides) ;

- 3 anticorps ont fourni un signal restreint aux fibres de type II, mais en se fixant de façon plus marquée sur certaines fibres II et beaucoup plus faible sur d'autres fibres II, aboutissant à la formation d'une mosaïque à l'observation des coupes transversales.

Ces trois derniers anticorps, à savoir S5 8H2, S5 7D4 et S5 11D6, ainsi que l'anticorps anti-I S5 7F10 ont été retenus.

L'analyse en Western-blot a montré que deux des anticorps anti-myosine rapide fournissant une mosaïque en immunofluorescence étaient des anticorps anti-II, et que le troisième était un anticorps anti-IIa, mais la mosaïque observée ne correspond pas à une distribution de fibres de type IIA et IIB, des fibres IIB étant positives. On peut interpréter cette distorsion par l'existence de fibres contenant à la fois des isoformes IIa et IIb correspondant à une maturation incomplète.

Les anticorps générés par ce projet constituent des outils utilisables en immunohistochimie. Ils sont en cours de test sur des myotubes issus de la régénération musculaire in vivo et sur des myoblastes en culture.

\section{E. BARREY, \\ J. P. VALETTE, M. JOUGLIN*}

\section{INRA Station de Génétique Quantitative et Appliquée, 78352 Jouy-en-Josas \\ * Ecole Nationale Vétérinaire d'Alfort, Laboratoire de Biomécanique, 94704 Maisons- Alfort Cedex}

\title{
Analyse de la composition en chaînes lourdes de myosine chez le cheval : application à la sélection du cheval de course
}

La performance du cheval de course dépend de plusieurs facteurs biologiques interdépendants : la respiration, la circulation sanguine, le métabolisme énergétique, les contractions musculaires, la locomotion et l'aptitude comportementale. Les propriétés contractiles et métaboliques des muscles propulseurs constituent l'un des facteurs limitants qui déterminent la force et l'endurance du cheval à la course (Barrey 1994). La force développée par les contractions musculaires dépend à la fois du nombre total et du type de fibres qui composent le muscle. Chez le cheval, il existe trois isoformes de la chaîne lourde de la myosine : la myosine lente (MHC I), et deux myosines rapides (MHC IIa et $\mathrm{MHC} \mathrm{IIb}$ ). Les fibres musculaires très riches en isoforme MHC IIb produisent des contractions plus puissantes mais moins durables. Une fibre musculaire peut être composée de plusieurs isoformes de la myosine (I+IIa ou IIa+IIb) et la myosine dominante détermine le type de fibre parmi les types lent (I) ou rapides (IIA, IIB) lorsqu'on les observe en histologie par la méthode de coloration ATPase (Rivero et al 1996). Le type de fibre rapide dénommée IIC en histolo- 
gie correspond en fait à un mélange des isoformes I et IIa de la myosine rapide qui donne une coloration intermédiaire. Contrairement à d'autres mammifères, le cheval adulte ne semble pas avoir de quatrième isoforme IIx de la chaîne lourde de la myosine. Cependant, la myosine MHC IIb du cheval a les mêmes propriétés de migration en électrophorèse que la myosine MHC IIx du rat, ce qui indiquerait qu'il s'agit peut-être de la même isoforme (Rivero et al 1996).

Afin de proposer des critères objectifs de sélection précoce des meilleurs chevaux de course, il paraissait intéressant d'estimer la composition en myosine de certains muscles propulseurs de la croupe. La composition en myosine de plusieurs muscles a été étudiée pour choisir un muscle qui soit à la fois représentatif du travail de propulsion en course et facile à prélever de manière standardisée. Le muscle fessier moyen (gluteus medius) réunit ces conditions et une étude de morphométrie par échographie a permis de définir deux points de prélèvement standardisés dans les parties dorsale et ventrale du muscle.

Pour envisager des études quantitatives sur des chevaux de valeur, il fallait disposer d'une technique de dosage sur micro prélèvement (10-30 mg) qui soit fiable, productive et peu coûteuse. L'emploi d'un anticorps monoclonal dirigé contre la myosine lente a permis de mettre au point une technique de dosage immunoenzymatique ELISA chez les bovins (Picard et al 1994) qui a été adaptée pour le cheval (Barrey et al 1995). Par la suite, l'utilisation d'un second anticorps dirigé contre les deux isoformes de la myosine rapide a amélioré la technique de dosage (Valette et al 1995).

Une première étude de la composition en mysosine du muscle fessier moyen chez les chevaux de race Anglo-Arabe a permis d'établir la relation avec les performances en compétition et d'estimer pour la première fois la composante génétique de ce caractère. Les meilleures performances étaient obtenues en course de galop (course sur un terrain plat d'environ $2000 \mathrm{~m}$ ) et en concours de saut d'obstacle (parcours d'obstacles) par des chevaux dont le muscle fessier moyen était plus riche en myosine rapide (Barrey et al 1997a). La différence de composition avec les chevaux ayant obtenu de moins bonnes performances était d'environ $6 \%$ dans les deux disciplines. L'héritabilité du pourcentage de myosine rapide dans le muscle fessier moyen (gluteus medius) et le muscle de la cuisse (gluteobiceps femoris) a été estimée, pour la première fois chez le cheval, à $13 \%$ dans la race AngloArabe, avec toutefois encore une large incertitude sur cette valeur (Erreur standard $=0,10$ ) (Barrey et al 1997b). Cette héritabilité semble plutôt faible par rapport à celle dans les autres espèces, car il existe une grande homogénéité de ce caractère musculaire dans cette race qui a déjà été sélectionnée pour la course. Ces premiers résultats ont montré l'intérêt de poursuivre ces travaux dans le but de faire des analyses en routine de la composition en
Figure 1. Identification du type de fibre musculaire par immunohistologie $(A, B$, $C)$ et histoenzymologie (D). Des coupes sériées du muscle latissimus dorsi d'un cheval ont été marquées par 3 anticorps monoclonaux dirigés respectivement contre les isoformes de la chaîne lourde de la myosine : SC 71 anti-MHC IIA (figure 1A), S5 $8 \mathrm{H} 2$ anti-MHC I+llb (figure 1B) et BF 35 anti-MHC I+llA. Pour comparaison, la coloration histologique classique est présentée figure $1 D$. Elle est basée sur la révélation de l'activité enzymatique des ATPases myofibrillaires après une incubation alkaline à $\mathrm{pH} 10,4$. Les types de fibres identifiés sont les suivants : 1 = fibre lente contenant $M H C I, 2=$ fibre rapide à dominante $M H C I A$, $3=$ fibre rapide à dominante $M H C I l b, 4=$ fibre rapide mixte de type IIC qui comprend les myosines MHC lla+llb. Ces coupes histologiques ont été obtenues avec la collaboration de J.L. Rivero (Université de Cordoba, Espagne).
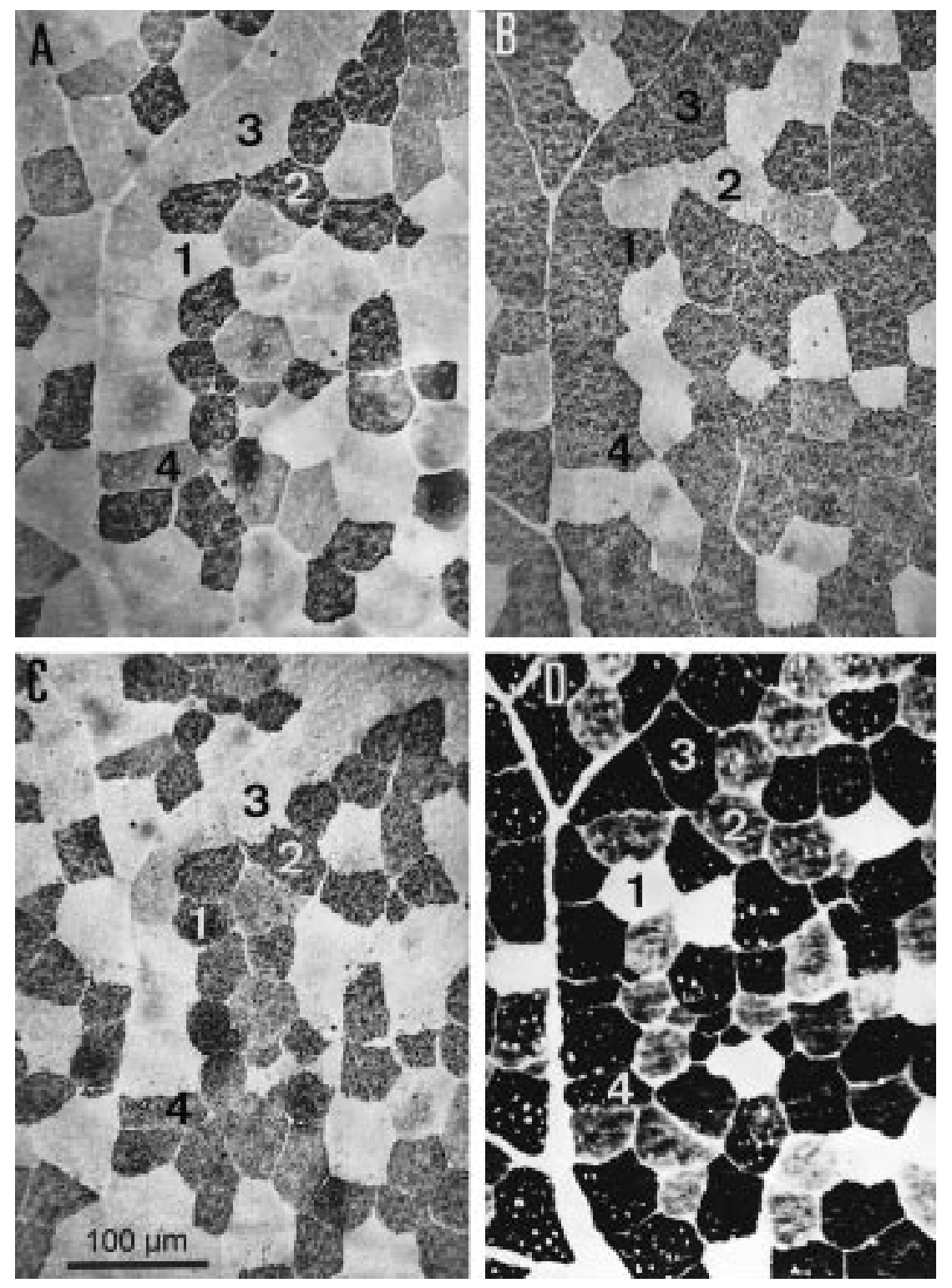

myosine pour aider à la sélection précoce des chevaux de course.

La faible variabilité phénotypique et génétique des races de chevaux de course incite à perfectionner la méthode de dosage afin de pouvoir distinguer la composition en deux isoformes de la myosine : MHC IIa et MHC IIb. En effet, les muscles plus riches en myosine IIa ont une meilleure aptitude à se contracter pendant une durée de quelques minutes tandis que ceux qui sont plus riches en myosine IIb peuvent développer des contractions plus 
puissantes pendant moins longtemps. Ainsi, le cheval de course (Pur sang) adapté à une distance d'environ $2000 \mathrm{~m}$ aurait des muscles plus riches en MHC IIa, tandis que le sprinter courant sur une courte distance $(<1000 \mathrm{~m})$ aurait davantage de MHC IIb et le cheval d'endurance (course de marathon de plus de $100 \mathrm{~km}$ ) aurait une proportion élevée de la somme des myosines MHC I et MHC IIa. Afin de doser ces différentes myosines, il fallait disposer d'anticorps monoclonaux spécifiques et améliorer la technique de dosage ELISA.

\section{Anticorps monoclonaux dirigés contre les MHC du muscle équin}

Tous les anticorps monoclonaux obtenus dans le projet Noé ont été testés dans un premier temps par la méthode ELISA en utilisant des muscles de référence, de composition déterminée préalablement par électrophorèse : masseter, cutaneus trunci, diaphragma, semitendinosus. Dans un deuxième temps, les anticorps retenus ont été testés par Western-blot, puis utilisés en immunohistologie pour typer les fibres sur des coupes sériées (J.L. Rivero 1997, données non publiées). En plus des anticorps produits par l'INRA, nous avons testé, selon la même procédure, des anticorps antiMHC IIa (SC 71), MHC IIb (BF F3) et MHC I (BA D5) spécifiques du muscle de rat, issus des travaux de Schiaffino et al (1989). Le tableau 1 résume les résultats obtenus avec les anticorps qui présentent un intérêt pour analyser le muscle du cheval. Les anticorps dirigés contre la MHC I sont réactifs chez le cheval, comme dans de nombreuses autres espèces. Par contre, la spécificité anti-MHC IIa de l'anticorps SC 71 semble limitée au rat et au cheval (figure 1). Aucun anticorps anti-MHC IIb n'a été identifié pour le muscle du cheval mais il existe un anticorps ( $\mathrm{S} 5 \mathrm{8H} 2$ ) ayant une double réactivité avec les myosines MHC I et MHC IIb. Ce marquage présente un intérêt pour l'identification des fibres musculaires par immunohistologie d'autant plus qu'il n'existe aucun autre anticorps susceptible de marquer spécifiquement les fibres IIb (figure 1).

\section{Détermination de la composition en MHC IIa par une technique ELISA}

L'anticorps monoclonal anti-MHC IIa (SC 71) nous a permis de perfectionner le dosage ELISA des isoformes de la myosine du muscle gluteus medius chez le cheval. De même que pour le dosage des myosines lente et rapide totales, une gamme étalon est établie pour déterminer la relation entre la densité optique du produit coloré final de la réaction immunoenzymologique et le pourcentage de myosine MHC IIa. Deux muscles de référence peuvent être utilisés pour établir cette courbe de calibration : le muscle gluteus medius ou le gluteobiceps qui a l'avantage d'être plus riche en MHC IIa (environ $60 \%$ ). La composition en myosine du muscle de référence est tout d'abord déterminée par électrophorèse (Talmadge et Roy 1993), puis différentes dilutions de la MHC IIa sont dosées par la technique ELISA afin de construire une droite de calibration qui met en relation la proportion en MHC IIa et la densité optique de la réaction immunoenzymatique. Ainsi, trois anticorps monoclonaux permettent de déterminer la composition en chaînes lourdes de la myosine MHC I, IIa et II totale et on en déduit par différence le pourcentage de MHC IIb.

\section{Conclusion}

L'obtention d'anticorps monoclonaux dirigés spécifiquement contre un type de chaîne lourde rapide MHC IIa et MHC IIb du cheval s'est avérée une opération difficile qui a nécessité la mise en œuvre de plusieurs méthodes d'analyse pour s'assurer de la spécificité des anticorps à tester (ELISA, immunohistologie, électrophorèse et Western-blot). Parmi les anticorps obtenus par l'INRA en collaboration avec Biocytex, un seul présente une réelle originalité pour l'espèce équine puisqu'il marque les myosines IIb et I en immunohistologie. D'autres anticorps, moins originaux puisqu'ils étaient déjà disponibles sur le marché, ont une affinité soit pour la

Tableau 1. Caractéristiques des anticorps monoclonaux du projet Noé et de Schiaffino et al (1989) ayant une affinité pour les isoformes des chaînes lourdes de la myosine chez le cheval.

\begin{tabular}{|l|l|c|c|c|}
\hline Anticorps & \multicolumn{1}{|c|}{ Origine } & Isotype & Immunohistologie & ELISA \\
\hline S5 8H2 & INRA ${ }^{(1)}$ & Ig G1 kappa & I+IIB & MHC I+IIb \\
S5 15F4 & INRA & Ig G1 kappa & IIA + IIB & MHC II \\
S4 10G9 & INRA & Ig G1 kappa & IIA + IIB & MHC II \\
S5 7E6 & INRA & Ig G1 kappa & I+IIA+IIB & MHC I+IIa+IIb \\
S4 9G11 & INRA & Ig M kappa & I & MHC I \\
SC 71 & Schiaffino et al $1989^{(2)}$ & Ig G1 kappa & IIA et hybrides IIC (I+IIA) & MHC IIa \\
& & & ou IIAB (IIA+IIB) & rien \\
BF F3 & Schiaffino et al 1989 & Ig M kappa & rien & MHC I \\
BA D5 & Schiaffino et al 1989 & Ig G2 b kappa & I & \\
\hline
\end{tabular}

(1) Anticorps conservés par Biocytex (Marseille, France).

(2) Anticorps conservés et commercialisés par DSM (Braunschweig, Allemagne). 
chaîne lourde de la myosine lente (MHC I), soit pour les chaînes lourdes des myosines rapides (MHC II totale). Un anticorps (SC 71) ayant une bonne spécificité pour la chaîne lourde de la myosine rapide de type IIa (MHC IIa) chez le cheval a été identifié parmi les anticorps monoclonaux obtenus par une autre équipe (Schiaffino et al 1989). Cet anticorps est utilisable pour les dosages par la technique ELISA et permettra de compléter nos connaissances sur la relation entre le profil musculaire et l'aptitude du cheval de course. Dans l'avenir, l'obtention d'un anticorps complémentaire dirigé contre la chaîne lourde de la myosine IIb permettrait d'améliorer la précision de la technique de dosage ELISA.

\section{Références}

Barrey E., 1994. Propriétés contractiles des fibres musculaires et performance physique chez le cheval. INRA Prod. Anim., 7, 41-53.

Barrey E., Valette J.P., Jouglin M., Picard B., Geay Y., Robelin J., 1995. Enzyme-linked immunosorbent assay for myosin heavy chains in horse. Reprod. Nutr. Dev., 35, 619-628.
Barrey E., Valette J.P., Jouglin M., Blouin C., Langlois B., 1997a. Relationship between the fast myosin heavy chain percentage of the gluteus medius muscle and the competitive performance in horses. 48th Annual Meeting of European Association for Animal Production, 25-28 August, Vienna, 156.

Barrey E., Valette J.P., Jouglin M., Blouin C., Langlois B., 1997b. Heritability of fast myosin heavy chain percentage of locomotor muscle in horses. 48th Annual Meeting of European Association for Animal Production, 25-28 August, Vienna, 160.

Picard B., Léger J.O.C., Robelin J., 1994. Quantitative determination of type $1 \mathrm{MHC}$ in bovine muscle with anti-myosine monoclonal antibodies. Meat Sci., $36,333-343$.

Rivero J.L., Talmadge R.J., Edgerton V.R., 1996. Myosin heavy chain isoforms in adult equine skeletal muscle : an immunohistochemical and electrophoretic study. Anat. Rec., 246, 185-194.

Schiaffino S., Gorza L., Sarore S., Saggin L., Ausoni S., Vianello M., Gundersen K., Lomo T., 1989. Three myosin heavy chain isoforms in type 2 skeletal muscle fibres. J. Muscl. Res. Cell. Motil., 10, 197-205.

Talmadge R., Roy R., 1993. Electrophoresis separation of rat skeletal muscle myosin heavy-chain isoforms. J. Appl. Physiol., 75, 2337-2340.

Valette J.P., Barrey E., Jouglin M., 1995. Slow myosin heavy chain content of muscles measured by ELISA. Equine vet. J., Suppl. 18, 248-251.

\section{Abstract}

Production and utilisation of monoclonal antibodies against myosin heavy chain isoforms in different species.

Monoclonal antibodies specific to myosin heavy chains (MHC) of different species of animals : bovine, pig, fish, chicken, turkey, horse have been produced. They have been tested by immunohistology on sections of skeletal muscle in bovine, pig, fish, chicken and turkey and by ELISA in horse. The different antibodies selected in the project allowed new applications for the study of skeletal muscle. In particular, two monoclonal antibodies can be used to classify by immunohistology the fibres IIA and IIB : one of them recognizing MHC I and IIb in bovine and horse and MHC I, IIb and IIx in pig, the other recognizing MHC IIa and IIx in pig. Some antibodies revealed heterogeneity in the myosin composition of fibres of white and red fish muscles as in the composition of fast myosin in chicken and turkey muscles without allowing a precise distinction of fibres IIA and IIB in the two species. More, in the rainbow trout, an antibody recognized more specifically the myosins of little fibres markers of the myogenesis de novo in the white muscle. However the obtention of specific antibodies against MHC IIa and IIb usable in particular in ELISA has not been achieved and remains an important necessity.

INRA Groupe Noé, Société Biocytex, 1998. Caractérisation des différents types de fibres musculaires dans plusieurs espèces : production et utilisation d'anticorps monoclonaux dirigés contre les chaînes lourdes de myosine rapide IIa et IIb. INRA Prod. Anim., 11, 145-163. 